\title{
Analysis of ECG Signal Denoising Using Wavelet Transform
}

\author{
Roshini T V $\mathbf{1}^{1}$, Shoukath Cherukat ${ }^{2}$, Seena $\mathbf{V}^{3}$ \\ Associate professor, Department of ECE, Vimaljyothi Engg College, Chemperi ${ }^{1}$ \\ Scientist - Engineer 'D', NIELIT, Calicut ${ }^{2}$ \\ M-Tech scholar, Department of ECE, Vimaljyothi Engg College, Chemperi ${ }^{3}$
}

\begin{abstract}
The electrocardiogram is a technique of recording bioelectric currents generated by the heart which is useful for diagnosing many cardiac diseases. The feature extraction and denoising of ECG are highly useful in cardiology. ECG is a non-stationary signal and it is used for the primary diagnosis of cardiac abnormalities like arrhythmia, myocardial infarction and conduction defects. But the ECG signal often contaminated by different noises. The ECG signal must be denoised to remove all the noises such as Additive White Gaussian noises. This paper deals with the analysis of ECG signal denoising using Wavelet Transform . Different ECG signals from MIT/BIH arrhythmia database are used with added AWG noise. Soft thresholding technique is employed in the signal and the result were evaluated using matlab. The Biorthogonal wavelet transform is applied on the different signal and the performance is evaluated in terms of PRD(percent root difference), PRD improvement (PRD i), SNR(signal to noise ratio),SNR improvement (SNRi)and compression ratio.
\end{abstract}

Keywords: ECG signal denoising, thresholding, Discrete wavelet transform, PRD and SNR.

\section{INTRODUCTION}

One of the main techniques widely used for diagnosing The earlier method of ECG signal analysis based on the heart disease is the electrocardiogram (ECG). time domain method[4]. But this is not sufficient to study Electrocardiogram[1] is the representation of electrical all the features of the ECG signal. To accomplish the activity of the heart recorded by number of electrodes frequency components Fast Fourier Transform (FFT) placed on the body surfaces. A normal ECG signal technique is applied. But this technique failed to provide waveform composed of $\mathrm{P}$ wave, $\mathrm{QRS}$ complex and $\mathrm{T}$ information regarding the exact location of the frequency wave. A small $\mathrm{U}$ wave is occasionally present. components. The immediate tool available for this purpose is the Short Term Fourier Transform(STFT). It is also

The detection of these parameter is important to analyse the ECG signal[2]. However ECG signal is a nonstationary signal, it is easy to interfere by the different noises while gathering and recording. Fig.1. shows a typical ECG waveform. The cardiac cycle begin with the $\mathrm{P}$ wave which corresponds to the atrial depolarization in the heart. The most striking part of the ECG is the QRS complex which is followed by $\mathrm{P}$ wave. The $\mathrm{T}$ wave follows the QRS complex and corresponds to the ventricular repolarization. The end point of the

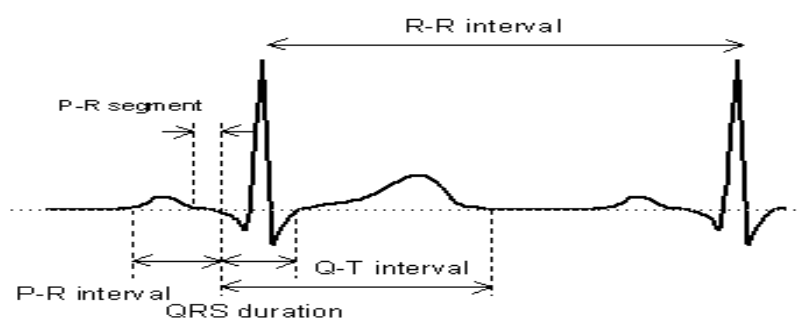

Fig.1. Normal ECG waveform

$\mathrm{T}$ wave represents the end of cardiac cycle [3] (assuming the absence of $u$ wave). The duration and amplitude level of particular parameter of the ECG is of great importance since it provide a measure of the state of the heart and indicate the certain cardiological condition. having a drawback that its time-frequency precision is not optimal. To overcome this drawback most simple and valuable technique is the wavelet transform. Recently wavelet transform has been widely used in signal and image processing due to the time-frequency localization characteristics. Wavelet analysis now become a common signal processing tool for analysing the ECG signal [5]. Analysis of ECG signal at desired section can be accomplished by decomposes the signal with various wavelet basis function that are well localized in time and frequency domain. This method is able to overcome the limitations of conventional methods.

\section{WAVELET TRANSFORM}

Wavelet transform involves representing a time function in terms of fixed building blocks, termed wavelets[7]. Wavelet transform has the ability of presenting the characteristics of signal in time field and frequency field[8]. Information characteristics of time is obtained through shifting the signal. The frequency characteristics of the signal is available through selected wavelet function [9]. Wavelet basis function have many families such as Haar, Daubechies, symlet etc, The wavelet transform at high frequency gives good time resolution and poor frequency resolution, while at low frequency the wavelet transform gives good frequency resolution and poor time resolution. 
1.1

2.1 Continuous wavelet transform

The continuous wavlet transform is a wavelet transform with a continous mother wavelet,continous detailed parameter and a discrete translation parameter. The continous wavelet transform is provided by equation 2.1 ,where $\mathrm{x}(\mathrm{t})$ is the signal to be analysed. $\Psi^{*}\left(\frac{t-b}{a}\right) \quad$ Is the conjugate of the mother wavelet function [10].

$$
x_{\omega}(a, b)=1^{\frac{1}{\sqrt{a}}} \int_{-\alpha}^{\alpha} x(t) \Psi^{*}\left(\frac{t-b}{a}\right) \mathbb{d} t
$$

1.2

\subsection{2 Discrete Wavelet Transform}

Discrete wavelet transform which is based on sub-band coding perform fast computation of wavelet transform. It is easy to implement and reduces the computation time and resources required. In $\mathrm{CW}$, the signal are analysed using a set of basis function which is relate to each other by simple scaling and translation parameter. In the case of DWT, a time scale representation of the digital signal is obtained using digital filtering technique. The signal to be analysed is passed through filters with different cutoff frequency and different scalars.

A general equation for DWT signal is written as

$$
X[a, b]=\sum_{n=-\alpha}^{\alpha} x(n) \phi_{a, b}(n)
$$

Where $\mathrm{x}(\mathrm{n})$ is the input signal to be analysed.

$$
\text { where } \quad \varnothing_{\mathrm{a}, \mathrm{b}}(\mathrm{n})=\frac{1}{\sqrt{a}} \phi\left(\frac{n-b}{a}\right)
$$

\section{ANALYSIS METHOD}

The various steps used for the performance analysis are discussed below.

\subsection{Database}

The first step in ECG signal processing is to obtain the signal from the data base available online. Database is MIT-BIH Arrhythmia database. Various ECG signal records are used for experiments and algorithm is tested from different record 101,102,103,104 and 105.The database is sampled at $360 \mathrm{~Hz}$ and the resolution of each sample is 11 bits/sample over $10 \mathrm{mV}$ range. The analysis was carried out using Matlab program which contains "wavelet toolbox". Fig.3.1 shows the ECG signal decomposition using biorthogonal wavelet filters for 101 records.

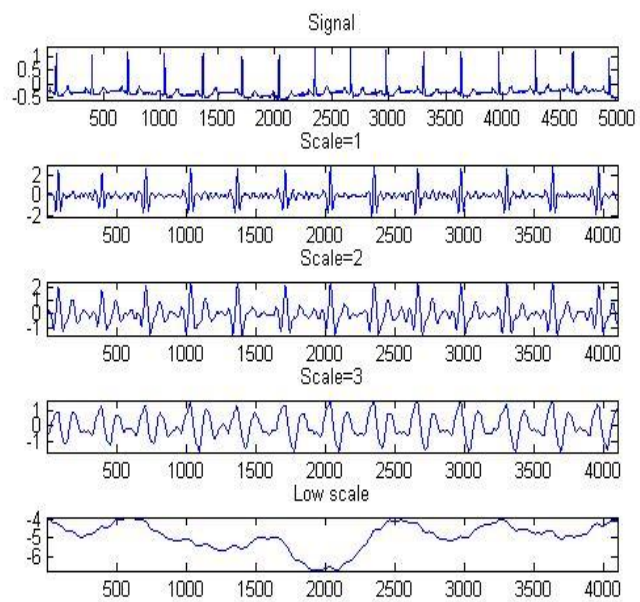

Fig.3.1.shows ECG decomposition for 101 records

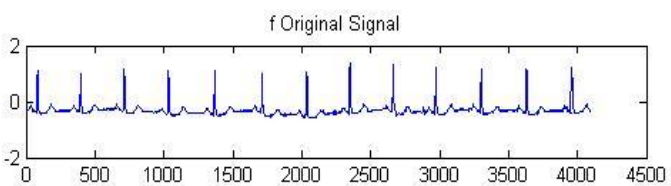

Noisy Signal
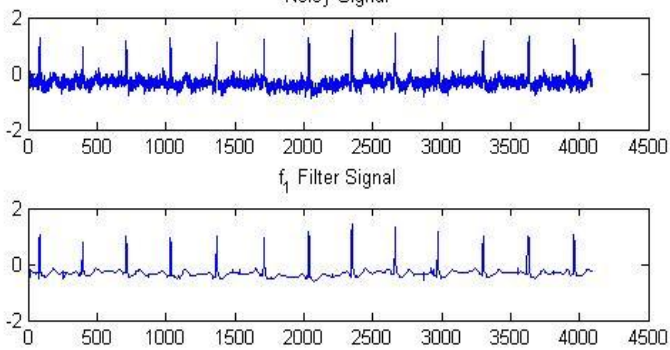

Fig 3.2 shows (a) original signal (b) noisy signal (c) filtered signal

\section{MEASURES OF PERFORMANCE}

4.1 Percent Root Mean Square Difference (PRD):

The most prominent distortion measure is the Percent Root Mean Square Difference(PRD) that is given by

$$
P R D=\sqrt{\frac{\sum_{n=1}^{N}[x[n]-\hat{x}[n]]^{2}}{\sum_{n=1}^{N}[x[n]]^{2}}} \times 100
$$

\subsection{Signal to Noise Ratio (SNR):}

Signal to noise ratio is a measure of power ratio between a signal and noise, It is expressed in terms of the

logarithmic decibel scale.

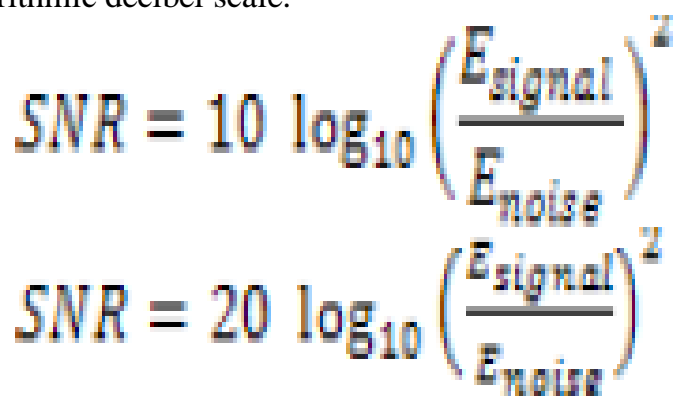

\subsection{Compression Ratio:}

The compression ratio is defined as the ratio of the number of bits representing the original signal to the number of bits required to store the compressed signal.

$$
C R=\frac{B_{\text {original }}}{B_{\text {compressed }}}
$$

\section{Where $\mathrm{B}_{\text {original }}-\quad$ Bit rate of the original signal \\ $\mathrm{B}_{\text {compressed- }}$ Bit rate of the compressed signal}

\section{RESULT AND DISCUSSION}

In this section we discussed on the result obtained with the experimental workdone. Five different data sets are used for the simulation purposes . These signal are considered as original signal or free of noisy signal. Fig.3.1 shows the result of decomposition of ECG signal using biorthogonal wavelet transform. Original ECG signal is 
added with Additive White Gaussian noise. Fig.3.2 shows the result of inverse wavelet transform to obtain denoised signal. The performance of the denoising is evaluated by the measures such as PRD, PRDi ,SNR, SNRi and CR. In the fig. 5.1. Plot of PRD and PRDi with different test cases. Fig.5.2. is the plot of SNR and SNRi with different test cases .Fig.5.2. represents the plot of compression ratio versus test cases. Form the result it can be analysed that for the data records 103 shows minimum PRD and maximum SNR.

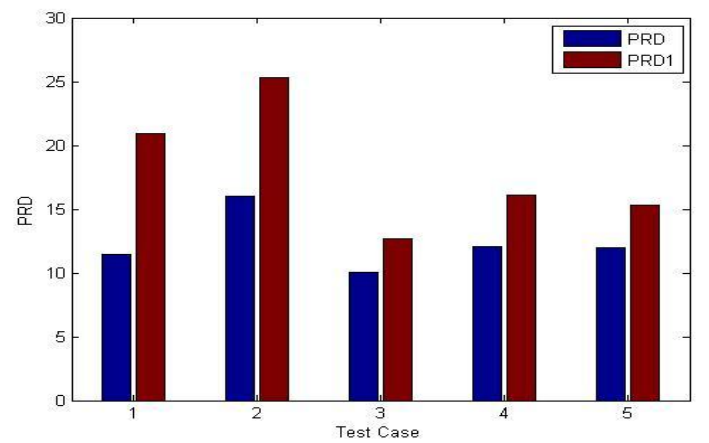

Fig.5.1. Plot of PRD and PRDi with different test cases.

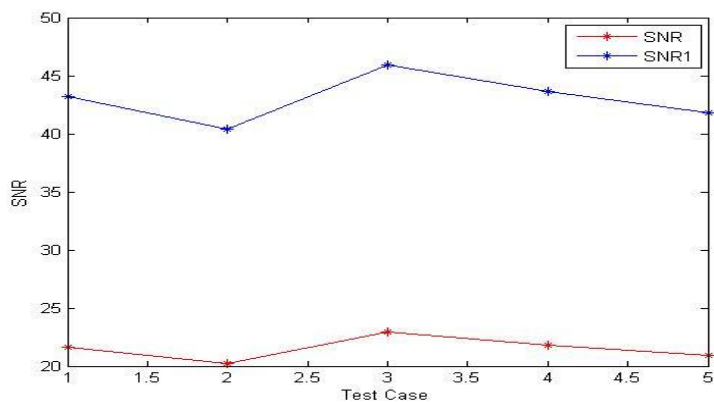

Fig.5.2 plot of SNR and SNRi with different test cases

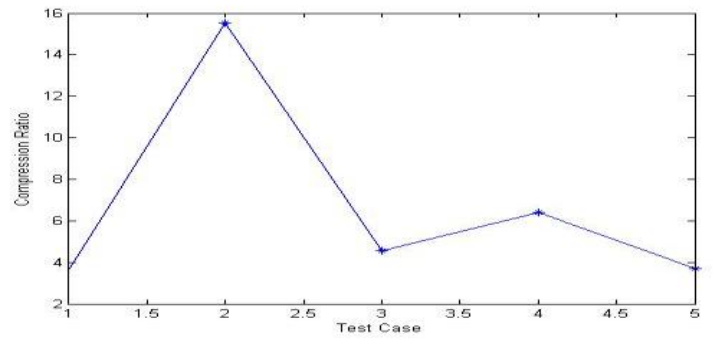

Fig.5.3. Plot of CR versus test cases

\section{CONCLUSION}

The techniques and methods for removing noise and interference is important in biomedical signal processing. Elimination of noise for ECG signal is a challenging problem by virtue of the fact that it may cause serious problem in the visual inspection of the signal. In this work we pointed out the advantage of using discrete wavelet transform for denoising of ECG signal. Biorthogonal wavelet transform is used here for the performance analysis. Wavelet transform is performed with different test cases and its performance in the different ECG signal is analysed.The future work focused on utilizing different thresholding methods based on a variety of wavelet that provides high accura

\section{REFERENCES}

[1] Prajakta S. Gokhala, "ECG signal denoising using Discrete Wavelet Transform for the removal of $50 \mathrm{~Hz}$ PLI noise",IJETAE,vol.2,issue 5 , march 2012.

[2] Jeong Yap Han,SuKyung Lee and Hony bacPark "Denoising of ECG using Translation Invarient Multiwavelt",Worldacademy of science, engineering and technology 2008.

[3] H.S. Narayana Murthy,M.Meenakshi,'ECG signal denoising and ischemic event feature extraction using Daubechies wavelet" International Journel of Computer application, Vol.67,No.2,april 2003.

[4] Seena.v,GraceJohn,"A review on denoising of ECG signal using wavelet transform",proc.on National conference,PIC2013.

[5] Pradnya B.Patil,Dr.Mahesh S. Chawan, "A Wavelet based method for denosing of Biomedical Signal", proc. on International conference on Pattern Recognitionand Information, march 2012.

[6] Ruqaiya Khanam,Syed Naseem,"Selection of Wavelets for evalusting SNR,PRD and CR of ECG signal",IJESIT,vol.2,January 2013

[7] Yun Yin,YuleHis "The Research on denoising Using Wavelet transform",IEEE 2011.

[8] Aaushi Kumari,Megha Bisht, "Analytical view of denoising methods in ECG signal", IJRCCT Vol.1,issue 5, October 2012.

[9]. I.Daubechies,"The wavelet transform,time- frequency Localization and signal analysis, IEEE Trans. inform.Theory 36(5) (1990), pp961-1005.

[10] MIT-BIHArrhythmia database,www.physionet.org. 